\title{
Galois and Pataki Connections on Generalized Ordered Sets
}

\section{Árpád Száz}

Department of Mathematics, University of Debrecen, H-4002 Debrecen, P.O. Box 400, Hungary

e-mail: szaz@science.unideb.hu

\section{Abstract}

In this paper, having in mind Galois and Pataki connections, we establish several basic theorems on increasingly seminormal and semiregular functions between gosets.

An ordered pair $X(\leq)=(X, \leq)$ consisting of a set $X$ and a relation $\leq$ on $X$ is called a goset (generalized ordered set).

A function $f$ of one goset $X$ to another $Y$ is called increasingly upper g-seminormal, for some function $g$ of $Y$ to $X$, if $f(x) \leq y$ implies $x \leq g(y)$.

While, the function $f$ is called increasingly upper $\varphi$-semiregular, for some function $\varphi$ of $X$ to itself, if $f(u) \leq f(v)$ implies $u \leq \varphi(v)$.

The increasingly lower seminormal (semiregular) functions are defined by the reverse implications. Moreover, a function is called increasingly normal (regular) if it is both increasingly upper and lower seminormal (semiregular).

The results obtained extend and supplement several former results of O. Ore and the present author on Galois and Pataki connections. Namely, the pairs $(f, g)$ and $(f, \varphi)$ may be called increasing Galois and Pataki connections if the function $f$ is increasingly $g$-normal and $\varphi$-regular, respectively.

Received: May 17, 2019; Accepted: July 1, 2019

2010 Mathematics Subject Classification: Primary 06A75, 06A15; Secondary 54E15, 54C60.

Keywords and phrases: ordered sets, Galois connections, uniform spaces, continuous relations.

The work of the author has been supported by the Hungarian Scientific Research Fund (OTKA) Grant $\mathrm{K}-111651$.

Copyright (C) 2019 Árpád Száz. This is an open access article distributed under the Creative Commons Attribution License, which permits unrestricted use, distribution, and reproduction in any medium, provided the original work is properly cited. 


\section{Introduction}

Ordered sets and Galois connections occur almost everywhere in mathematics [8]. They allow of transposing problems and results from one world of our imagination to another one.

In [28], having in mind a terminology of Birkhoff [1, p. 1], an ordered pair $X(\leq)=(X, \leq)$ consisting of a set $X$ and a relation $\leq$ on $X$ is called a goset (generalized ordered set).

In particular, a goset $X(\leq)$ is called a proset (preordered set) if the relation $\leq$ is reflexive and transitive. And, a proset is $X(\leq)$ called a poset (partially ordered set) if the relation $\leq$ is in addition antisymmetric.

In [35], motivated by an ingenious observation of Schmidt [20, p. 209] a function $f$ of one goset $X$ to another $Y$ is called increasingly upper g-seminormal, for some function $g$ of $Y$ to $X$, if $f(x) \leq y$ implies $x \leq g(y)$ for all $x \in X$ and $y \in Y$.

While, motivated by a fundamental definition of Pataki [17, p. 160], the function $f$ is called increasingly upper $\varphi$-semiregular, for some function $\varphi$ of $X$ into itself, if $f(u) \leq f(v)$ implies $u \leq \varphi(v)$ for all $u, v \in X$.

Thus, if $f$ is increasingly upper $g$-seminormal, then by taking $\varphi=g \circ f$, we can at once see that $f(u) \leq f(v) \Rightarrow u \leq g(f(v)) \Rightarrow u \leq(g \circ f)(v) \Rightarrow u \leq \varphi(v)$ for all $u, v \in X$. Therefore, $f$ is increasingly upper $\varphi$-semiregular.

The increasingly lower seminormal (semiregular) functions are defined by the reverse implications. Moreover, a function is called increasingly normal (regular) if it is both increasingly upper and lower seminormal (semiregular).

Now, in particular, the pairs $(f, g)$ and $(f, \varphi)$ may also be naturally called increasing Galois and Pataki connections between the gosets $X$ and $Y$ if the function $f$ is increasingly $g$-normal and $\varphi$-regular, respectively.

Moreover, we can at once see that a function $f$ of one goset $X$ to another $Y$ is increasingly lower $\Delta_{X}$-semiregular if and only it is increasing in the usual sense that $u \leq v$ implies $f(u) \leq f(v)$ for all $u, v \in X$. 
In this respect, it is also worth mentioning that if $f$ is an increasingly $\varphi$-regular function of one proset $X$ to another $Y$, then $f$ is increasing and $\varphi$ is a closure operation on $X$ such that $\varphi \circ f \leq f \leq \varphi \circ f$. (See [33].)

Here, a function $\varphi$ of a goset $X$ to itself is called a closure operation if it is increasing, extensive and lower semiidempotent in the sense that $x \leq \varphi(x)$ and $\varphi(\varphi(x)) \leq \varphi(x)$ for all $x \in X$. Thus, $\varphi$ is idempotent if $X$ is a poset.

The importance of regular functions is also apparent from the fact that a function $\varphi$ of a proset $X$ to itself is a closure operation if and only if $\varphi$ is increasingly $\varphi$-regular. That is, $u \leq \varphi(v)$ is equivalent to $\varphi(u) \leq \varphi(v)$ for all $u, v \in X$.

In [33], we have also proved that a function $f$ of one proset $X$ to another $Y$ is increasingly $\varphi$-regular if and only if $\varphi$ is a closure operation on $X$ such that $\varphi(u) \leq \varphi(v)$ is equivalent to $f(u) \leq f(v)$ for all $u, v \in X$.

Several interesting characterizations of increasingly regular and normal functions have also been established in [31,35]. Moreover, some closely related results for increasing functions and closure operations have also been proved in [40].

Now, by improving and extending some of these results, we shall, for instance, prove that for a function $f$ of a sup-complete proset $X$ to an arbitrary one $Y$, the following assertions are equivalent:

(1) $f$ is increasingly normal,

(2) $f[\sup (A)] \subseteq \sup (f[A])$ for all $A \subseteq X$,

(3) $f$ is increasing and $\sup \left(\operatorname{Int}_{f}(y)\right) \subseteq \operatorname{Int}_{f}(y)$ for all $y \in Y$,

(4) $f$ is increasing and $\max \left(\operatorname{Int}_{f}(y)\right)=\sup \left(\operatorname{Int}_{f}(y)\right)$ for all $y \in Y$,

(5) $f$ is increasing and $f[\sup (A)] \subseteq \mathrm{lb}(\mathrm{ub}(f[A]))$ for all $A \subseteq X$,

(6) $f$ is increasing and $f[\mathrm{lb}(\mathrm{ub}(A))] \subseteq \mathrm{lb}(\mathrm{ub}(f[A]))$ for all $A \subseteq X$.

Here, by definition, we have $\operatorname{Int}_{f}(y)=\{x \in X: f(x) \leq y\}$ for all $y \in Y$. Moreover, it is noteworthy that if $X$ and $Y$ are supposed to be only arbitrary gosets, then (1) already implies the second statement of (6). 
In [40], for a function $f$ of one goset $X$ to another, we have only prove that $f$ is increasing if and only if $f[\mathrm{ub}(A)] \subseteq \mathrm{ub}(f[A])$ for all $A \subseteq X$. Moreover, if $f$ is increasing, then $\mathrm{lb}(\mathrm{ub}(f[A])) \subseteq \mathrm{lb}(f[\mathrm{ub}(A)])$ for all $A \subseteq X$.

At the end of this paper, we shall also offer some generalizations of increasingly seminormal functions on posets to relations on relator spaces of the form $(X, Y)(\mathcal{R})$, where $X$ and $Y$ are sets and $\mathcal{R}$ is a family of relations on $X$ to $Y$.

\section{A Few Basic Facts on Relations}

A subset $F$ of a product set $X \times Y$ is called a relation on $X$ to $Y$. If in particular $F \subseteq X^{2}$, with $X^{2}=X \times X$, then we may simply say that $F$ is a relation on $X$. In particular, $\Delta_{X}=\{(x, x): x \in X\}$ is called the identity relation on $X$.

If $F$ is a relation on $X$ to $Y$, then by the above definitions we can also state that $F$ is a relation on $X \cup Y$. However, for several purposes, the latter view of the relation $F$ would be quite unnatural.

If $F$ is a relation on $X$ to $Y$, then for any $A \subseteq X$ and $x \in X$ the sets $F[A]=\{y \in Y: \exists x \in A:(x, y) \in F\}$ and $F(x)=F[\{x\}]$ are called the images of $A$ and $x$ under $F$, respectively.

Moreover, the sets $D_{F}=\{x \in X: F(x) \neq \varnothing\}$ and $R_{F}=F[X]$ are called the domain and range of $F$, respectively. If in particular $D_{F}=X$, then we say that $F$ is a relation of $X$ to $Y$, or that $F$ is a non-partial relation on $X$ to $Y$.

In particular, a relation $f$ on $X$ to $Y$ is called a function if for each $x \in D_{f}$ there exists $y \in Y$ such that $f(x)=\{y\}$. In this case, by identifying singletons with their elements, we may simply write $f(x)=y$ instead of $f(x)=\{y\}$.

Moreover, a function $\star$ of $X$ to itself is called a unary operation on $X$. While, a function $*$ of $X^{2}$ to $X$ is called a binary operation on $X$. And, for any $x, y \in X$, we usually write $x^{\star}$ and $x * y$ instead of $\star(x)$ and $*((x, y))$.

If $F$ is a relation on $X$ to $Y$, then a function $f$ of $D_{F}$ to $Y$ is called a selection of $F$ if 
$f \subseteq F$, i.e., $f(x) \in F(x)$ for all $x \in D_{F}$. Thus, the Axiom of Choice can be briefly expressed by saying that every relation has a selection.

For any relation $F$ on $X$ to $Y$, we may naturally define two set-valued functions, $F^{\diamond}$ of $X$ to $\mathcal{P}(Y)$ and $F^{\diamond}$ of $\mathcal{P}(X)$ to $\mathcal{P}(Y)$, such that $F^{\diamond}(x)=F(x)$ for all $x \in X$ and $F^{\diamond}(A)=F[A]$ for all $A \subset X$.

Functions of $X$ to $\mathcal{P}(Y)$ can be identified with relations on $X$ to $Y$. While, functions of $\mathcal{P}(X)$ to $\mathcal{P}(Y)$ are usually more powerful tools than relations on $X$ to $Y[38,46]$. However, they are frequently less flexible.

If $F$ is a relation on $X$ to $Y$, then $F=\bigcup_{x \in X}\{x\} \times F(x)$. Therefore, the values $F(x)$, where $x \in X$, uniquely determine $F$. Thus, a relation $F$ on $X$ to $Y$ can also be naturally defined by specifying $F(x)$ for all $x \in X$.

For instance, the complement relation $F^{c}$ can be naturally defined such that $F^{c}(x)=F(x)^{c}=Y \backslash F(x)$ for all $x \in X$. The latter notation will not cause confusions, since thus we also have $F^{c}=X \times Y \backslash F$.

Quite similarly, the inverse relation $F^{-1}$ can be naturally defined such that $F^{-1}(y)=\{x \in X: y \in F(x)\}$ for all $y \in Y$. Thus, the operations $c$ and -1 are compatible in the sense $\left(F^{c}\right)^{-1}=\left(F^{-1}\right)^{c}$.

Moreover, if in addition $G$ is a relation on $Y$ to $Z$, then the composition relation $G \circ F$ can be naturally defined such that $(G \circ F)(x)=G[F(x)]$ for all $x \in X$. Thus, we also have $(G \circ F)[A]=G[F[A]]$ for all $A \subseteq X$.

While, if $G$ is a relation on $Z$ to $W$, then the box product relation $F \otimes G$ can be naturally defined such that $(F \otimes G)(x, z)=F(x) \times G(z)$ for all $x \in X$ and $z \in Z$. Thus, we have $(F \otimes G)[A]=G \circ A \circ F^{-1}$ for all $A \subseteq X \times Z[36]$.

Hence, by taking $A=\{(x, z)\}$, and $A=\Delta_{Y}$ if $Y=Z$, one can see that the box and composition products are actually equivalent tools. However, the box product can be immediately defined for arbitrary families of relations. 
Now, a relation $R$ on $X$ may be briefly called reflexive if $\Delta_{X} \subseteq R$, and transitive if $R \circ R \subseteq R$. Moreover, $R$ may be briefly called symmetric if $R^{-1} \subseteq R$, and antisymmetric if $R \cap R^{-1} \subseteq \Delta_{X}$.

Thus, a reflexive and transitive (symmetric) transitive relation may be called a preorder (tolerance) relation. And, a symmetric (antisymmetric) preorder relation may be called an equivalence (partial order) relation.

For instance, for $A \subseteq X$, Pervin's relation $P_{A}=A^{2} \cup A^{c} \times X$ is a preorder relation on $X$ [32]. While, for a pseudo-metric $d$ on $X$ and $r>0$, Weil's surrounding $B_{r}^{d}=\left\{(x, y) \in X^{2}: d(x, y)<r\right\}$ is a tolerance relation on $X$.

Note that $S_{A}=R_{A} \cap R_{A}^{-1}=R_{A} \cap R_{A^{c}}=A^{2} \cap\left(A^{c}\right)^{2}$ is already an equivalence relation on $X$. And, more generally if $\mathcal{A}$ is a cover (partition) of $X$, then $S_{\mathcal{A}}=\bigcup_{A \in \mathcal{A}} A^{2}$ is a tolerance (equivalence) relation on $X$.

Now, for any relation $R$ on $X$, we may also naturally define $R^{0}=\Delta_{X}$ and $R^{n}=R \circ R^{n-1}$ if $n \in \mathbb{N}$. Moreover, we may naturally define $R^{\infty}=\bigcup_{n=0}^{\infty} R^{n}$. Thus, $R^{\infty}$ is the smallest preorder relation on $X$ containing $R$ [12] .

\section{A Few Basic Facts on Generalized Ordered Sets}

According to [28], an ordered pair $X(\leq)=(X, \leq)$, consisting of a set $X$ and a relation $\leq$ on $X$, will be called generalized ordered set, or an ordered set without axioms. And, we shall usually write $X$ in place of $X(\leq)$.

In the sequel, a generalized ordered set $X(\leq)$ will, for instance, be called reflexive if the relation $\leq$ is reflexive. Moreover, the generalized ordered set $X^{\prime}\left(\leq^{\prime}\right)=X\left(\leq^{-1}\right)$ will be called the dual of $X(\leq)$.

Having in mind the terminology of Birkhoff [1, p. 1], a generalized ordered set may be briefly called a goset. Moreover, a preordered (partially ordered) set may be called a proset (poset). 
Thus, every set $X$ is a poset with the identity relation $\Delta_{X}$. Moreover, $X$ is a proset with the universal relation $X^{2}$. And every subfamily of the power set $\mathcal{P}(X)$ of $X$ is a poset with the ordinary set inclusion $\subseteq$.

The usual definitions on posets can be naturally extended to gosets [28]. (And, even to arbitrary relator spaces [27] which include ordered sets [7], context spaces [10], and uniform spaces [9] as the most important particular cases.)

For instance, for any subset $A$ of a goset $X$, we may naturally define

$$
\begin{array}{rr}
\operatorname{lb}(A)=\{x \in X: & \forall a \in A: \quad x \leq a\}, \\
\operatorname{ub}(A)=\{x \in X: & \forall a \in A: \quad a \leq x\}, \\
\min (A)=A \cap \operatorname{lb}(A), & \max (A)=A \cap \mathrm{ub}(A), \\
\inf (A)=\max (\operatorname{lb}(A)), & \sup (A)=\min (\operatorname{ub}(A)) .
\end{array}
$$

In the sequel, by identifying singletons with their elements, we shall, for instance, write $\mathrm{ub}(x)$ instead of $\mathrm{ub}(\{x\})$ for all $x \in X$. Thus, we have

$$
\mathrm{ub}(x)=\leq(x)=[x,+\infty[=\{y \in X: x \leq y\}
$$

for all $x \in X$.

Now, as an immediate of the corresponding definitions, we can state

Theorem 2.1. For any subset $A$ of a goset $X$, we have
(1) $\mathrm{lb}(A)=\bigcap_{a \in A} \operatorname{lb}(a)$,
(2) $\mathrm{ub}(A)=\bigcap_{a \in A} \mathrm{ub}(a)$.

Hence, it is clear that in particular we also have

Corollary 2.2. If $X$ is a goset, then

(1) $\operatorname{lb}(\varnothing)=X$ and $\mathrm{ub}(\varnothing)=X$,

(2) $\mathrm{lb}(B) \subseteq \mathrm{lb}(A)$ and $\mathrm{ub}(B) \subseteq \mathrm{ub}(A)$ for all $A \subseteq B \subseteq X$.

By using Theorem 2.1, we can also easily prove the following

Theorem 2.3. If $\Phi$ is a unary operation on $\mathcal{P}(X)$, for some set $X$, such that $\Phi(A)=\bigcap_{a \in A} \Phi(a)$ for all $A \subseteq X$, then there exists a relation $\leq$ on $X$ such that $\Phi=\mathrm{lb}_{\leq}\left(\Phi=\mathrm{ub}_{\leq}\right)$. 
However, it is now more important to note that we also have the following

Theorem 2.4. For any two subsets $A$ and $B$ of a goset $X$, we have

$$
A \subseteq \mathrm{lb}(B) \Leftrightarrow B \subseteq \mathrm{ub}(A)
$$

Proof. By the corresponding definitions, each of the above inclusions is equivalent to the property that $a \leq b$ for all $a \in A$ and $b \in B$, which can be briefly expressed by writing that $A \times B \subseteq \leq$. (That is, $A \in \mathrm{Lb}_{\leq}(B)$ or $B \in \mathrm{Ub}_{\leq}(A)$ by [27])

Remark 2.5. The above theorem shows that

$$
\mathrm{lb}(A) \subseteq^{\prime} B \Leftrightarrow A \subseteq \mathrm{ub}(B)
$$

for all $A, B \subset X$.

Therefore, the set-functions $\mathrm{lb}$ and $\mathrm{ub}$ form a Galois connection between the poset $\mathcal{P}(X)$ and its dual in the sense of [7, Definition 7.23], suggested by Schmidt's reformulation [20, p. 209] of Ore's definition of Galois connexions [16].

Remark 2.6. Hence, by taking $\Phi=\mathrm{ub} \circ \mathrm{lb}$, we can easily see that

$$
\mathrm{lb}(A) \subseteq^{\prime} \mathrm{lb}(B) \Leftrightarrow A \subseteq \Phi(B)
$$

for all $A, B \subset X$.

Therefore, the set-functions $\mathrm{lb}$ and $\Phi$ form a Pataki connection between the poset $\mathcal{P}(X)$ and its dual in the sense of [31, Remark 3.8] suggested by a fundamental unifying work of Pataki [17] on the basic refinements of relators studied each separately by the present author in [24] .

By [33], the letter fact implies that $\mathrm{lb}=\mathrm{lb} \circ \Phi$, and the function $\Phi$ is a closure operation on the poset $\mathcal{P}(X)$ in the sense of [1, p. 111]. By an observation, attributed to Dedekind by Erné $[8$, p. 50], this is equivalent to the requirement that the function $\Phi$ with itself form a Pataki connection between the poset $\mathcal{P}(X)$ and itself.

\section{Some Further Results on Gosets}

Concerning minima and maxima, and infima and suprema, one can easily prove the following theorems. 
Theorem 3.1. For any subset $A$ of a goset $X$, we have

(1) $\min (A)=\{x \in A: A \subseteq \mathrm{ub}(x)\}, \quad$ (2) $\max (A)=\{x \in A: A \subseteq \mathrm{lb}(x)\}$.

Remark 3.2. By this theorem, for instance, we may also naturally define

$$
\mathrm{ub}^{*}(A)=\{x \in X: A \cap \mathrm{ub}(x) \subseteq \mathrm{lb}(x)\} .
$$

Thus, $\max ^{*}(A)=A \cap \mathrm{ub}^{*}(A)$ is just the family of all maximal elements of $A$.

Theorem 3.3. For any subset $A$ of a goset $X$, we have
(1) $\inf (A)=\operatorname{lb}(A) \cap \mathrm{ub}(\mathrm{lb}(A))$,
(2) $\sup (A)=\mathrm{ub}(A) \cap \mathrm{lb}(\mathrm{ub}(A))$.

Theorem 3.4. For any subset $A$ of a goset $X$, we have

(1) $\inf (A)=\sup (\operatorname{lb}(A))$,

(3) $\min (A)=A \cap \inf (A)$,

Theorem 3.5. Under the notation $\Phi=$ min, max, inf, or sup, for any subset $A$ of an antisymmetric goset $X$, we have $\operatorname{card}(\Phi(A)) \leq 1$.

Remark 3.6. Conversely, one can also easily see that if $X$ is a reflexive goset such that $\operatorname{card}(\Phi(A)) \leq 1$ for all $A \subseteq X$, with $\operatorname{card}(A)=2$, then $X$ is antisymmetric.

In [29], by using the notation $\mathcal{L}=\{A \subseteq X: A \subseteq \operatorname{lb}(A)\}$, we have first proved that a reflexive goset $X$ is antisymmetric if and only if $\operatorname{card}(A) \leq 1$ for all $A \in \mathcal{L}$.

Definition 3.7. A goset $X$ is called inf-complete (sup-complete) if $\inf (A) \neq \varnothing$ $(\sup (A) \neq \varnothing)$ for all $A \subseteq X$.

Remark 3.8. Quite similarly, a goset $X$ may, for instance, be also naturally called min-complete if $\min (A) \neq \varnothing$ for all nonvoid subset $A$ of $X$.

Thus, the set $\mathbb{N}$ of all natural numbers is min-, but not inf-complete. While, the extended real line $\overline{\mathbb{R}}=\mathbb{R} \cup\{-\infty,+\infty\}$ is inf-, but not min-complete.

Now, as an immediate consequence of Theorem 3.4, we can state the following straightforward extension of [1, Theorem 3, p. 112]. 
Theorem 3.9. For a goset $X$, the following assertions are equivalent:

(1) X is inf-complete,

(2) $X$ is sup-complete.

Remark 3.10. Similar equivalences of several modified inf- and sup-completeness properties of gosets have been established in [4] and [3].

Definition 3.11. A goset $X$ is called linear if for any $u, v \in X$, with $u \neq v$, we have either $u \leq v$ or $v \leq u$.

Remark 3.12. If $X$ is a goset, then for any $u, v \in X$ we write $u<v$ if both $u \leq v$ and $u \neq v$.

Therefore, if the goset $X$ is linear, then for any $u, v \in X$, with $u \neq v$, we actually have either $u<v$ or $v<u$.

Moreover, as a consequence of the corresponding definitions, we can also state

Theorem 3.13. For a goset $X$, the following assertions are equivalent:

(1) $X$ is reflexive and linear,

(2) for any $u, v \in X$, we have either $u \leq v$ or $v \leq u$,

(3) $\min (A) \neq \varnothing(\max (A) \neq \varnothing)$ for all $A \subseteq X$ with $1 \leq \operatorname{card}(A) \leq 2$.

Hence, it is clear that in particular we also have

Corollary 3.14. If $X$ is a min-complete (max-complete) goset, then $X$ is reflexive and linear.

The importance of reflexive, linear, and antisymmetric gosets is apparent from the following

Theorem 3.15. (1) If $X$ is an antisymmetric goset, then $u<v$ implies $v \nless u$ for all $u, v \in X$,

(2) While, if $X$ is a reflexive and linear goset, then $u \not v$ implies $v<u$ for all $u, v \in X$.

Proof. To check (2), note that if $u \leq v$, then by Theorem 3.13 we have $v \leq u$. Moreover, by the reflexivity of $X$, we also have $v \neq u$. Therefore, we also have $v<u$. 
Now, as an immediate consequence of this theorem, we can also state the following very particular Galois-type connection.

Corollary 3.16. If $X$ is a reflexive, linear and antisymmetric goset, then for any $u, v \in X$ we have $u<v$ if and only if $v \leq u$.

Remark 3.17. If $X\left(\leq_{X}\right)$ is a goset and $Y \subseteq X$, then by taking $\leq_{Y}=\leq_{X} \cap Y^{2}$ we can at once see that $Y\left(\leq_{Y}\right)$ is also a goset which inherits several basic properties of the original goset $X\left(\leq_{X}\right)$.

Moreover, concerning subgosets, we can also easily prove the following

Theorem 3.18. If $X$ is a goset and $Y \subseteq X$, then for any $A \subseteq Y$ we have
(1) $\min _{Y}(A)=\min _{X}(A)$,
(2) $\min _{Y}(A)=\min _{X}(A)$,
(3) $\mathrm{lb}_{Y}(A)=\mathrm{lb}_{X}(A) \cap Y$,
(4) $\mathrm{ub}_{Y}(A)=\mathrm{ub}_{X}(A) \cap Y$,
(5) $\inf _{X}(A) \cap Y \subseteq \inf _{Y}(A)$,
(6) $\sup _{X}(A) \cap Y \subseteq \sup _{Y}(A)$.

Proof. To check (5), note that if $\alpha \in \inf _{X}(A)$, then by Theorem 3.3 we have $\alpha \in \mathrm{lb}_{X}(A)$ and $\alpha \in \mathrm{ub}_{X}\left(\mathrm{lb}_{X}(A)\right)$. Hence, if $\alpha \in Y$ also holds, by using (3) we can see that $\alpha \in \operatorname{lb}_{Y}(A)$.

Moreover, if $v \in \mathrm{lb}_{Y}(A)$, then by using (3) we can see that $v \in Y$ and $v \in \mathrm{lb}_{X}(A)$. Hence, since $\alpha \in \mathrm{ub}_{X}\left(\mathrm{lb}_{X}(A)\right)$, we can infer that $v \leq \alpha$. This shows that $\alpha \in \mathrm{ub}_{X}\left(\mathrm{lb}_{Y}(A)\right)$. Hence, since $\alpha \in Y$, by using (3) we can already infer that $\alpha \in \mathrm{ub}_{Y}\left(\mathrm{lb}_{Y}(A)\right)$. Thus, by Theorem 3.3, $\alpha \in \sup _{Y}(A)$ also holds.

Remark 3.19. In connection with (5), Tamás Glavosits, my $\mathrm{PhD}$ student, showed that the corresponding equality need not be true even if $X$ is finite poset.

For this, he took $X=\{a, b, c, d\}, \quad Y=X \backslash\{b\}, A=Y \backslash\{a\}$, and considered the preorder $\leq$ on $X$ generated by the relation $R=\{(a, b),(b, c),(b, d)\}$.

Thus, he could at once see that $\inf _{Y}(A)=\max \left(\operatorname{lb}_{Y}(A)\right)=\max (\{a\})=\{a\}$, but $\inf _{X}(A)=\max \left(\operatorname{lb}_{X}(A)\right)=\max (\{a, b\})=\{b\}$, and thus $\inf _{X}(A) \cap Y=\varnothing$. 


\section{Increasing Functions of One Goset to Another}

Increasing functions are usually called isotone, monotone, or order-preserving in algebra. Moreover, in [7, p. 186] even the extensive maps are called increasing. However, we prefer to use a terminology of analysis [21, p. 128].

Definition 4.1. If $f$ is a function of one goset $X$ to another $Y$, then we say that:

(1) $f$ is increasing if $u \leq v$ implies $f(u) \leq f(v)$ for all $u, v \in X$,

(2) $f$ is strictly increasing if $u<v$ implies $f(u)<f(v)$ for all $u, v \in X$.

Remark 4.2. Quite similarly, the function $f$ may be called decreasing if $u \leq v$ implies $f(v) \leq f(u)$ for all $u, v \in X$.

Thus, we can note that $f$ is a decreasing function of $X$ to $Y$ if and only if it is an increasing function of $X$ to the dual $Y^{\prime}$ of $Y$.

Therefore, the study of decreasing functions can be traced back to that of the increasing ones. In [40], by proving the following statements, we have shown that almost the same is true in connection with the strictly increasing ones.

Theorem 4.3. For a function $f$ of one goset $X$ to another $Y$, the following assertions hold:

(1) $f$ is strictly increasing if $f$ is injective and increasing,

(2) $f$ is injective if $X$ is linear and $f$ is strictly increasing.

Remark 4.4. Note that if in particular $Y$ is reflexive, then the strict increasingness of $f$ also implies the increasingness of $f$.

In [40], concerning strictly increasing functions, we have also proved

Theorem 4.5. If $f$ is a strictly increasing function of a linear goset $X$ onto an antisymmetric one $Y$, then $f^{-1}$ is a strictly increasing function of $Y$ onto $X$.

However, it is now more important to note that, by [40], we also have

Theorem 4.6. For a function $f$ of one goset $X$ to another $Y$, the following assertions are equivalent:

(1) $f$ is increasing, 
(2) $f[\mathrm{ub}(x)] \subseteq \mathrm{ub}(f(x))$ for all $x \in X$,

(3) $f[\mathrm{ub}(A)] \subseteq \mathrm{ub}(f[A])$ for all $A \subseteq X$.

Remark 4.7. Note that $f$ is an increasing function of $X$ to $Y$ if and only if it is an increasing function of $X^{\prime}$ to $Y^{\prime}$.

Therefore, in the above theorem we may write $\mathrm{lb}$ in place of $\mathrm{ub}$. However, because of Theorem 3.3 and Corollary 2.2, we cannot write sup instead of ub.

Despite this, in [40], we could also easily prove the following

Theorem 4.8. For a function $f$ of a reflexive goset $X$ to an arbitrary one $Y$, the following assertions are equivalent:

(1) $f$ is increasing,

(2) $f[\max (A)] \subseteq \mathrm{ub}(f[A])$ for all $A \subseteq X$,

(3) $f[\max (A)] \subseteq \max (f[A])$ for all $A \subseteq X$,

(4) $f[\max (A)] \subseteq \mathrm{ub}(f[A])$ for all $A \subseteq X$ with $\operatorname{card}(A) \leq 2$.

Proof. To prove the implication (1) $\Rightarrow(3)$, note that if (1) holds, then by the definition of maximum and Theorem 4.6 we have

$$
\begin{aligned}
f[\max (A)] & =f[A \cap \mathrm{ub}(A)] \subseteq f[A] \cap f[\mathrm{ub}(A)] \\
& \subseteq f[A] \cap \mathrm{ub}(f[A])=\max (f[A])
\end{aligned}
$$

for all $A \subseteq X$ even if $X$ is not assumed to be reflexive.

Now, by using this theorem, we can also easily prove

Corollary 4.9. If $f$ is a function on a reflexive goset $X$ to an arbitrary one $Y$ such that

$$
f[\sup (A)] \subseteq \sup (f[A])
$$

for all $A \subseteq X$ with $\operatorname{card}(A) \leq 2$, then $f$ is already increasing.

Proof. If $A$ is as above, then by Theorems 3.4 and 3.3 we have

$$
f[\max (A)] \subseteq f[\sup (A)] \subseteq \sup (f[A]) \subseteq \mathrm{ub}(f[A]) .
$$

Therefore, by Theorem $4.8, f$ is increasing. 


\section{Closure and Interior Operations on Gosets}

According to [40], we shall now also use the following

Definition 5.1. If $\varphi$ is a unary operation on a goset $X$, then we say that:

(1) $\varphi$ is extensive (intensive) if $\Delta_{X} \leq \varphi\left(\varphi \leq \Delta_{X}\right)$,

(2) $\varphi$ is upper (lower) semiidempotent if $\varphi \leq \varphi^{2}\left(\varphi^{2} \leq \varphi\right)$.

Remark 5.2. Moreover, $\varphi$ may be naturally called upper (lower) semiinvolutive if $\varphi^{2}$ is extensive (intensive). That is, $\Delta_{X} \leq \varphi^{2}\left(\varphi^{2} \leq \Delta_{X}\right)$.

Remark 5.3. In this respect, it is also worth noticing that $\varphi$ is upper (lower) semiidempotent if and only if its restriction to its range is extensive (intensive). Therefore, if $\varphi$ is extensive (intensive), then $\varphi$ is upper (lower) semiidempotent.

The importance of extensive functions is also quite obvious from the following theorem of [40].

Theorem 5.4. If $\varphi$ is a strictly increasing operation on a min-complete, antisymmetric goset $X$, then $\varphi$ is extensive.

Remark 5.5. To feel the importance of extensive operations, it is also worth noticing that if $\varphi$ is an extensive operation on an antisymmetric goset, then each maximal element $x$ of $X$ is already a fixed point of $\varphi$ in the sense that $\varphi(x)=x$.

This fact has also been strongly emphasized by Brøndsted [5]. Moreover, fixed point theorems for extensive maps (which are sometimes called expansive, progressive, increasing, or inflationary) were also proved in [13], [7, p. 188], and [15].

The following theorem of [40] shows that, in contrast to the injective, increasing functions the inverse of an injective, extensive operation need not be extensive.

Theorem 5.6. If $\varphi$ is an injective and extensive operation on antisymmetric goset $X$ such that $X=\varphi[X]$ and $\varphi^{-1}$ is also extensive, then $\varphi=\Delta_{X}$.

From this theorem, by using Theorems 4.5 and 5.4, we can immediately derive 
Corollary 5.7. If $\varphi$ is a strictly increasing operation on a min-complete, antisymmetric goset $X$ such that $X=\varphi[X]$, then $\varphi=\Delta_{X}$.

In general, the idempotent operations are quite different from the both upper and lower semiidempotent ones. However, we may still naturally use the following

Definition 5.8. An increasing, extensive (intensive) operation is called a preclosure (preinterior) operation. And, a lower semiidempotent (upper semiidempotent) preclosure (preinterior) operation is called a closure (interior) operation.

Moreover, an extensive (intensive) lower semiidempotent (upper semiidempotent) operation is called a semiclosure (semiinterior) operation. While, an increasing and upper (lower) semiidempotent operation is called an upper (lower) semimodification operation.

Remark 5.9. Thus, $\varphi$ is, for instance, an interior operation on a goset $X$ if and only if it is a closure operation on the dual $X^{\prime}$ of $X$.

Concerning closure operations, in [40] we have, for instance, proved the following two theorems.

Theorem 5.10. If $\varphi$ is a closure operation on a sup-complete, transitive, and antisymmetric goset $X$, then for any $A \subseteq X$, we have

$$
\varphi(\sup (A))=\varphi(\sup (\varphi[A]))
$$

Hence, it is clear that in particular we also have the following

Corollary 5.11. Under the above conditions, for any $A \subseteq X$, we have

$$
\varphi(\sup (A))=\sup (\varphi[A]) \text { if and only if } \sup (\varphi[A])=\varphi(\sup (\varphi[A])) .
$$

Theorem 5.12. If $\varphi$ is a closure operation on a transitive goset $X$, and $Y=\varphi[A]$, then for any $A \subseteq Y$ we have

$$
\varphi\left[\sup _{X}(A)\right] \subseteq \sup _{Y}(A)
$$

Hence, by using Definition 3.7 and Theorem 3.5, we can immediately derive

Corollary 5.13. If in addition to the above conditions, $X$ is sup-complete and antisymmetric, then for any $A \subseteq Y$ we have

$$
\sup _{Y}(A)=\varphi\left(\sup _{X}(A)\right)
$$


Remark 5.14. Surprisingly, the corresponding infimum properties of closure operations are much simpler, and do not require the transitivity of $X$.

\section{The Induced Order and Interior Relations}

Definition 6.1. For a function $f$ of a set $X$ to a goset $Y$, we define a relation $\operatorname{Ord}_{f}$ on $X$ such that

$$
\operatorname{Ord}_{f}(u)=\{v \in X: f(u) \leq f(v)\}
$$

for all $u \in X$. The relation $\operatorname{Ord}_{f}$ will be called the natural order induced by $f$.

Remark 6.2. Thus, under the notation $\leq_{f}=\operatorname{Ord}_{f}$, for any $u, v \in X$, we have $u \leq_{f} v$ if and only if $f(u) \leq f(v)$.

Therefore, as an immediate consequence of the corresponding definitions, we can state the following

Theorem 6.3. If $f$ is a function of a set $X$ to a goset $Y$, then $\operatorname{Ord}_{f}$ is the largest relation on $X$ making $f$ to be increasing.

Proof. If $\leq$ is a relation on $X$ making $f$ to be increasing, then $u \leq v$ implies $f(u) \leq f(v)$ implies $u \leq_{f} v$ for all $u, v \in X$. Therefore, $\leq \subseteq \leq_{f}$, and thus $\leq \subseteq$ Ord $_{f}$ is also true.

Several further basic properties of the relation Ord $_{f}$ have been proved in [35] .

For instance, as some immediate consequences of some slightly more general results, we have established the following two theorems.

Theorem 6.4. If $f$ is a function of a set $X$ to a goset $Y$, then

(1) $\operatorname{Ord}_{f}$ is a preorder on $X$ if $Y$ is a proset,

(2) $\operatorname{Ord}_{f}$ is a partial order on $X$ if $f$ is injective and $Y$ is a poset.

Theorem 6.5. A function $f$ of a goset $X$ to a proset $Y$ is increasing if and only if any one of the following assertions holds:
(1) Ord $_{f}$ is decreasing,
(2) Ord $_{f}$ is ascending valued,
(3) $\operatorname{Ord}_{f}^{-1}$ is increasing,
(4) $\operatorname{Ord}_{f}^{-1}$ is descending valued. 
Remark 6.6. A relation $F$ on a goset $X$ to a set $Y$ is called increasing if the induced set-valued function $F^{\diamond}$ is increasing. That is, $u \leq v$ implies $F(u) \subseteq F(v)$ for all $u, v \in X$.

While, a relation $F$ on a set $X$ to a goset $Y$ is called ascending valued if the function $F^{\diamond}$ is ascending valued. That is, $v \in F(u)$ and $v \leq w$ imply $w \in F(u)$ for all $u \in X$ and $w \in Y$.

By [35], a relation $F$ on a goset $X$ to a set $Y$ is increasing if and only if its inverse is ascending valued. And dually, a relation $F$ on a set $X$ to a goset $Y$ is descending valued if and only if its inverse is decreasing.

Definition 6.7. For any function $f$ of a set $X$ to a goset $Y$, we define a relation Int $f$ on $Y$ to $X$ such that

$$
\operatorname{Int}_{f}(y)=\{x \in X: f(x) \leq y\}
$$

for all $y \in Y$. The relation $\operatorname{Int}_{f}$ will be called the proximal interior induced by $f$.

Remark 6.8. Note that if in particular $f$ is a function of a power set $\mathcal{P}(X)$ to a goset $Y$, then by identifying singletons with their elements, we may also naturally define int $_{f}(y)=X \cap \operatorname{Int}_{f}(y)$ for all $y \in Y$. Thus, int $f$ is a relation on $Y$ to $X$ which may be called the topological interior induced by $f$.

Note also that if in particular $R$ is a relation on $X$ to $Y$, then in accordance with our former definitions, we have $\operatorname{Int}_{R}=\mathrm{Int}_{R^{\diamond}}$ and $\operatorname{int}_{R}=$ int $_{R^{\diamond}}=$ Int $_{R^{\diamond}}$. Therefore, we may also naturally define $\operatorname{Ord}_{R}=\operatorname{Ord}_{R^{\diamond}}$ and $\operatorname{ord}_{R}=\operatorname{Ord}_{R^{\diamond}}$. Thus, for instance, for any $u, v \in X$ we have $v \in \operatorname{ord}_{R}(u)$ if and only if $R(u) \subseteq R(v)$.

Concerning the relation $\mathrm{Int}_{f}$, in [35] we have, for instance, proved the following theorems.

Theorem 6.9. If $f$ is a function of a set $X$ to a goset $Y$, then

$$
\operatorname{Ord}_{f}=\left(\operatorname{Int}_{f} \circ f\right)^{-1}=f^{-1} \circ \operatorname{Int}_{f}^{-1} .
$$

Theorem 6.10. If $f$ is a function of a set $X$ to a goset $Y$, then for any $y \in Y$ and $x \in X$ we have 


$$
\operatorname{Int}_{f}(y)=f^{-1}[\operatorname{lb}(y)] \quad \text { and } \quad \operatorname{Int}_{f}^{-1}(x)=\mathrm{ub}(f(x)) \text {. }
$$

Remark 6.11. In this respect, it is also worth noticing that $y \in \operatorname{ub}\left(f\left[\operatorname{Int}_{f}(y)\right]\right)$.

Namely, by the definition of $\operatorname{Int}_{f}$, for every $x \in \operatorname{Int}_{f}(y)$, we have $f(x) \leq y$.

Theorem 6.12. If $f$ is a function of a set $X$ to a transitive goset $Y$, then

(1) Int $_{f}$ is increasing,

(2) $\operatorname{Int}_{f}^{-1}$ is ascending valued.

Remark 6.13. If in particular $X$ is also a goset and $f$ is increasing, then we can also state that

(1) Int $_{f}^{-1}$ is decreasing, (2) $\mathrm{Int}_{f}$ is descending valued.

However, in view of the corresponding results of Section 4, it is now more important to note that following theorem is also true.

Theorem 6.14. If $f$ is a function of one goset $X$ to another $Y$ such that

$$
f[\sup (A)] \subseteq \mathrm{lb}(\mathrm{ub}(f[A]))
$$

for all $A \subseteq X$, then

$$
\max \left(\operatorname{Int}_{f}(y)\right)=\sup \left(\operatorname{Int}_{f}(y)\right)
$$

for all $y \in Y$.

Proof. If $y \in Y$, then by Theorem 3.4 we have

$$
\max \left(\operatorname{Int}_{f}(y)\right) \subseteq \sup \left(\operatorname{Int}_{f}(y)\right) .
$$

Therefore, we need actually prove only the converse inclusion.

For this, note that if $x \in \sup \left(\operatorname{Int}_{f}(y)\right)$, then by the assumed property of $f$ we have

$$
f(x) \in f\left[\sup \left(\operatorname{Int}_{f}(y)\right)\right] \subseteq \mathrm{lb}\left(\operatorname{ub}\left(f\left[\operatorname{Int}_{f}(y)\right]\right)\right) .
$$

Moreover, by Remark 6.11, we also have $y \in u b\left(f\left[\operatorname{Int}_{f}(y)\right]\right)$. Therefore, we necessarily have $f(x) \leq y$, and thus $x \in \operatorname{Int}_{f}(y)$. Hence, by Theorem 3.4, we can see that

$$
x \in \operatorname{Int}_{f}(y) \cap \sup \left(\operatorname{Int}_{f}(y)\right)=\max \left(\operatorname{Int}_{f}(y)\right) .
$$

Therefore, $\sup \left(\operatorname{Int}_{f}(y)\right) \subseteq \max \left(\operatorname{Int}_{f}(y)\right)$, and thus the required equality is also true. 
Remark 6.15. Note that, by Theorem 3.4, for a subset $A$ of the goset $X$ we have $\max (A)=\sup (A)$ if and only if $\sup (A) \subseteq A$.

\section{Galois Type Connections between Gosets}

In [35], slightly extending the ideas of Ore [16], Schmidt [20, p. 209], Blyth and Janowitz [2, p. 11], and the present author [33] on Galois connections, residuated mappings, and increasingly normal functions, we have introduced the following

Definition 7.1. If $f$ is a function on one goset $X$ to another $Y$ and $g$ is a function of $Y$ to $X$, then we say that:

(1) $f$ is increasingly upper $g$-seminormal if $f(x) \leq y$ implies $x \leq g(y)$ for all $x \in X$ and $y \in Y$,

(2) $f$ is increasingly lower g-seminormal if $x \leq g(y)$ implies $f(x) \leq y$ for all $x \in X$ and $y \in Y$.

Remark 7.2. Now, the function $f$ may be naturally called increasingly g-normal if it is both increasingly upper and lower $g$-seminormal.

Moreover, a function $f$ of $X$ to $Y$ may, for instance, be naturally called increasingly normal if it is increasingly $g$-normal for some function $g$ of $Y$ to $X$.

Later, we shall see that the increasingly normal functions are closely related to the increasing ones. Therefore, in accordance with Remark 4.2, a function $f$ of $X$ to $Y$ may, for instance, be naturally called decreasingly normal if it is increasing normal as a function of $X$ to the dual $Y^{\prime}$ of $Y$.

In this respect, it is also worth mentioning that in [35] we have proved the following simple dualization principle.

Theorem 7.3. If $f$ is an increasingly upper (lower) g-seminormal function of one goset $X$ to another $Y$, then $g$ is an increasingly lower (upper) $f$-seminormal function of $Y^{\prime}$ to $X^{\prime}$.

Proof. If $f$ is increasingly upper $g$-seminormal, then by the corresponding definitions it is clear that

$$
y \leq^{\prime} f(x) \Rightarrow f(x) \leq y \Rightarrow x \leq g(y) \Rightarrow g(y) \leq^{\prime} x
$$


for all $y \in Y$ and $x \in X$. Therefore, $g$ is increasingly lower $f$-seminormal as a function of $Y^{\prime}$ to $X^{\prime}$.

Corollary 7.4. If $f$ is an increasingly g-normal function of one goset $X$ to another $Y$, then $g$ is an increasingly $f$-normal function of $Y^{\prime}$ to $X^{\prime}$.

Remark 7.5. By Theorem 7.3, the properties of the functions $g$ and $f \circ g$ can be immediately derived from those of $f$ and $g \circ f$. However, it is sometimes more convenient to apply a direct proof.

In [35], having in mind the properties of the function $\varphi=g \circ f$, and slightly extending the ideas of Pataki [17] and the present author [33], we have also introduced the following

Definition 7.6. If $f$ is a function on one goset $X$ to another $Y$ and $\varphi$ is a unary operation on $X$, then we say that:

(1) $f$ is increasingly upper $\varphi$-semiregular if $f(u) \leq f(v)$ implies $u \leq \varphi(v)$ for all $u, v \in X$,

(2) $f$ is increasingly lower $\varphi$-semiregular if $u \leq \varphi(v)$ implies $f(u) \leq f(v)$ for all $u, v \in X$.

Remark 7.7. Now, the function $f$ may be naturally called increasingly $\varphi$-regular if it is both increasingly upper and lower $\varphi$-semiregular.

Moreover, a function $f$ of $X$ to $Y$ may, for instance, be naturally called increasingly regular if it is increasingly $\varphi$-regular for some unary operation $\varphi$ on $X$.

Analogously to Remark 7.2, a function $f$ of $X$ to $Y$ may, for instance, be naturally called decreasingly regular if it is increasingly regular as a function of $X$ to $Y^{\prime}$. Unfortunately, now we do not have a counterpart of Theorem 7.3.

However, to clarify the relationship between normal and regular functions, in [35] we have proved the following two theorems.

Theorem 7.8. If $f$ is an increasingly upper (lower) g-seminormal function of one goset $X$ to another $Y$, then $\varphi=g \circ f$ is a unary operation on $X$ such that $f$ is increasingly upper (lower) $\varphi$-semiregular. 
Corollary 7.9. If $f$ is an increasingly g-normal function of one goset $X$ to another $Y$, then $\varphi=g \circ f$ is a unary operation on $X$ such that $f$ is increasingly $\varphi$-regular.

Theorem 7.10. If $f$ is an increasingly upper (lower) $\varphi$-semiregular function of one goset $X$ onto another $Y$ and $g$ is a function of $Y$ to $X$ such that $\varphi=g \circ f$, then $f$ is increasingly upper (lower) g-seminormal.

Proof. Suppose that $x \in X$ and $y \in Y$. Then, since $Y=f[X]$, there exists $v \in X$ such that $y=f(v)$.

Now, if $f$ is increasingly upper $\varphi$-semiregular, then we can easily see that $f(x) \leq y \Rightarrow f(x) \leq f(v) \Rightarrow x \leq \varphi(v) \Rightarrow x \leq(g \circ f)(v) \Rightarrow x \leq g(f(v)) \Rightarrow x \leq g(y)$.

Therefore, $f$ is increasingly upper $g$-seminormal too.

Corollary 7.11. If $f$ is an increasingly $\varphi$-regular function of one goset $X$ onto another $Y$ and $g$ is a function of $Y$ to $X$ such that $\varphi=g \circ f$, then $f$ is increasingly g-normal.

Remark 7.12. By Theorem 7.8, it is clear that several properties of the increasingly normal functions can be immediately derived from those of the increasingly regular ones. Therefore, the latter ones have to study before the former ones.

Moreover, from Theorem 7.10, we can see that the increasing regular functions are still less general objects than the increasingly normal ones. Later, we shall see that they are strictly between closure operations and increasingly normal functions.

\section{Some Basic Properties of Increasingly Semiregular Functions}

In [35], as some immediate consequences of the corresponding definitions, we have also proved the following theorems and their corollaries.

Theorem 8.1. If $f$ is an increasingly upper $\varphi$-semiregular function of an arbitrary goset $X$ to a reflexive one $Y$, then $\varphi$ is extensive, and thus it is also upper semiidempotent.

Proof. Because of the reflexivity of $Y$, for any $x \in X$, we have $f(x) \leq f(x)$. Hence, by using the assumed semiregularity of $f$, we can already infer that $x \leq \varphi(x)$. Therefore, $\varphi$ is extensive, and thus by Remark 5.3 it is also upper semiidempotent. 
Corollary 8.2. If $f$ is an increasingly upper $\varphi$-semiregular function of an arbitrary goset $X$ to a reflexive one $Y$ such that $f$ is increasing, then $f \leq f \circ \varphi$.

Theorem 8.3. If $f$ is an increasingly lower $\varphi$-semiregular function of a reflexive goset $X$ to an arbitrary one $Y$, then $f \circ \varphi \leq f$.

Corollary 8.4. If $f$ is an increasingly $\varphi$-regular function of a reflexive goset $X$ to a reflexive, antisymmetric one $Y$ such that $f$ is increasing, then $f=f \circ \varphi$.

Theorem 8.5. If $f$ is an increasingly $\varphi$-regular function of a reflexive goset $X$ to a transitive one $Y$, then $\varphi$ is lower semiidempotent.

Proof. By Theorem 8.3, we have $f \circ \varphi \leq f$. Hence, by using the corresponding definitions, we can infer that $f \circ \varphi^{2} \leq f \circ \varphi$. Now, by the transitivity of $Y$, it is clear that $f \circ \varphi^{2} \leq f$ also holds. Therefore, for any $x \in X$, we have $f\left(\varphi^{2}(x)\right) \leq f(x)$. Hence, by using the increasing upper $\varphi$-semiregularity of $f$, we can already infer that $\varphi^{2}(x) \leq \varphi(x)$.

Corollary 8.6. If $f$ is an increasingly $\varphi$-regular function of a reflexive goset $X$ to a proset $Y$, then $\varphi$ is a semiclosure operation on $X$.

Theorem 8.7. If $f$ is an increasingly $\varphi$-regular function of a transitive goset $X$ to a reflexive one $Y$, then $f$ is increasing, and thus $f \leq f \circ \varphi$ also holds.

Proof. By Theorem 8.1, we have $x \leq \varphi(x)$ for all $x \in X$. Therefore, if $u, v \in X$ such that $u \leq v$, then by the inequality $v \leq \varphi(v)$ and the transitivity of $X$, we also have $u \leq \varphi(v)$. Hence, by using the increasing lower $\varphi$-semiregularity of $f$, we can infer that $f(u) \leq f(v)$. Therefore, $f$ is increasing, and thus by Corollary 8.2 the required inequality is also true.

Corollary 8.8. If $f$ is an increasingly $\varphi$-regular function of a proset $X$ to a reflexive, antisymmetric goset $Y$, then $f=f \circ \varphi$.

Theorem 8.9. If $f$ is an injective, increasingly $\varphi$-regular function of a reflexive goset $X$ to a reflexive, antisymmetric goset $Y$ such that either $f$ is increasing or $X$ is transitive, then $\varphi=\Delta_{X}$. 
Theorem 8.10. If $f$ is an increasingly upper $\Delta_{X}$-semiregular function of an antisymmetric goset $X$ to a reflexive goset $Y$, then $f$ is injective.

Corollary 8.11. If $f$ is an increasingly $\varphi$-regular function $f$ of one reflexive, antisymmetric goset $X$ to another $Y$ such that either $f$ is increasing or $X$ is transitive, then $f$ injective if and only if $\varphi=\Delta_{X}$.

Theorem 8.12. If $f$ is an increasingly $\varphi$-regular function of one proset $X$ to another $Y$, then $\varphi$ is a closure operation on $X$.

Proof. By Corollary 8.6, we need only show that $\varphi$ is also increasing. For this, note that if $u, v \in X$ such that $u \leq v$, then by Theorem 8.7 we have $f(u) \leq f(v)$. Moreover, by Theorem 8.3 , we have $f(\varphi(u)) \leq f(u)$. Thus, by the transitivity of $Y$, we also have $f(\varphi(u)) \leq f(v)$. Hence, by using the increasing upper $\varphi$-semiregularity of $f$, we can already infer that $\varphi(u) \leq \varphi(v)$.

Theorem 8.13. If $\varphi$ is an extensive operation on a transitive goset $X$, then $\varphi$ is increasingly upper $\varphi$-semiregular.

Corollary 8.14. A unary operation $\varphi$ on a proset $X$ is extensive if and only if it is increasingly upper $\varphi$-semiregular.

Theorem 8.15. If $\varphi$ is a lower semimodification operation on a transitive goset $X$, then $\varphi$ is increasingly lower $\varphi$-semiregular.

Corollary 8.16. If $\varphi$ is a closure operation on transitive goset $X$, then $\varphi$ is increasingly $\varphi$-regular.

Theorem 8.17. For a unary operation $\varphi$ on a proset $X$, the following assertions are equivalent:

(1) $\varphi$ is a closure operation, (2) $\varphi$ is increasingly $\varphi$-regular,

(3) there exists an increasingly $\varphi$-regular function $f$ of $X$ to a proset $Y$.

Corollary 8.18. For a function $f$ of one proset $X$ to another $Y$ and a unary operation $\varphi$ on $X$, the following assertions are equivalent: 
(1) $f$ is increasingly $\varphi$-regular,

(2) $\varphi$ is a closure operation and $\operatorname{Ord}_{\varphi}=\operatorname{Ord}_{f}$.

Remark 8.19. Note that, by the corresponding definitions, $\operatorname{Ord}_{\varphi}=\operatorname{Ord}_{f}$ means only that, for any $u, v \in X$, we have $\varphi(u) \leq \varphi(v)$ if and only if $f(u) \leq f(v)$.

\section{Some Basic Properties of Increasingly Seminormal Functions}

From the corresponding results of Section 8, by using Theorem 7.8 and its corollary, we can immediately derive the following assertions.

Theorem 9.1. If $f$ is an increasingly upper g-seminormal function of an arbitrary goset $X$ to a reflexive one $Y$, then $g \circ f$ is extensive and upper semiidempotent.

Corollary 9.2. If $f$ is an increasingly upper g-seminormal function of an arbitrary goset $X$ to a reflexive one $Y$ such that $f$ is increasing, then $f \leq f \circ g \circ f$.

Theorem 9.3. If $f$ is an increasingly lower g-seminormal function of a reflexive goset $X$ to an arbitrary one $Y$, then $f \circ g \circ f \leq f$.

Corollary 9.4. If $f$ is an increasingly g-normal function of a reflexive goset $X$ to a reflexive, antisymmetric one $Y$ such that $f$ is increasing, then $f=f \circ g \circ f$.

Theorem 9.5. If $f$ is an increasingly g-normal function of a reflexive goset $X$ to a transitive one $Y$, then $g \circ f$ is lower semiidempotent.

Corollary 9.6. If $f$ is an increasingly g-normal function of a reflexive goset $X$ to a proset $Y$, then $g \circ f$ is a semiclosure operation on $X$.

Theorem 9.7. If $f$ is an increasingly g-normal function of a transitive goset $X$ to a reflexive one $Y$, then $f$ is increasing and $f \leq f \circ g \circ f$.

Corollary 9.8. If $f$ is an increasingly $g$-normal function of a proset $X$ to a reflexive, antisymmetric goset $Y$, then $f=f \circ g \circ f$.

Theorem 9.9. If $f$ is an injective, increasingly g-normal function of a reflexive goset $X$ to a reflexive, antisymmetric one $Y$ such that either $f$ is increasing or $X$ is transitive, then $g \circ f=\Delta_{X}$, and thus $g$ is onto $X$. 
Now, by using Corollaries 9.4 and 9.8, we can also easily prove

Theorem 9.10. If $f$ is an increasingly $g$-normal function of a reflexive goset $X$ onto a reflexive, antisymmetric one $Y$ such that either $f$ is increasing or $X$ is transitive, then $f \circ g=\Delta_{Y}$, and thus $g$ is injective.

Proof. By Corollaries 9.4 and 9.8, we have $f(g(f(x)))=f(x)$ for all $x \in X$. Hence, by using $Y=f(X)$, we can infer that $f(g(y))=y$ for all $y \in Y$.

Moreover, from Theorem 8.12, by Corollary 7.9, it is clear that we also have

Theorem 9.11. If $f$ is an increasingly g-normal function of one proset $X$ to another $Y$, then $g \circ f$ is a closure operation.

Now, as a partial converse to Theorems 9.1 and 9.7 , we can also prove

Theorem 9.12. If $f$ is an arbitrary function of a transitive goset $X$ to an arbitrary one $Y$ and $g$ is an increasing function of $Y$ to $X$ such that $g \circ f$ is extensive, then $f$ is increasingly upper g-seminormal.

Proof. If $x \in X$ and $y \in Y$ such that $f(x) \leq y$, then by the increasingness of $g$ we also have $(g \circ f)(x) \leq g(y)$. Moreover, by the extensiveness of the operation $g \circ f$, we also have $x \leq(f \circ g)(x)$. Hence, by the transitivity of $X$, it follows that $x \leq g(y)$. Therefore, the required assertion is also true.

From this theorem, by Theorem 9.1, it is clear that in particular we also have

Corollary 9.13. If $f$ is an arbitrary function of a transitive goset $X$ to a reflexive one $Y$ and $g$ is an increasing function of $Y$ to $X$, then $g \circ f$ is extensive if and only if $f$ is increasingly upper g-seminormal.

Finally, we note that as some immediate consequences of Theorems 9.1 and 9.12, we can also state the following two theorems.

Theorem 9.14. If $\varphi$ is an increasingly upper $\varphi$-seminormal operation on a reflexive goset $X$, then $\varphi$ is upper semiinvolutive.

Theorem 9.15. If $\varphi$ is an increasing, upper semiinvolutive operation on a transitive goset $X$, then $\varphi$ is increasingly upper $\varphi$-seminormal. 
Hence, by Theorem 9.7, it is clear that in particular we also have

Corollary 9.16. A unary operation $\varphi$ on a proset $X$ is increasingly upper $\varphi$-seminormal if and only if it is increasing and upper semiinvolutive.

\section{Some Further Properties of Increasingly Seminormal Functions}

From the corresponding results of Section 9, by using Theorem 7.3 and its corollary, we can immediately derive the following assertions.

Theorem 10.1. If $f$ is an increasingly lower $g$-seminormal function of a reflexive goset $X$ to an arbitrary one $Y$, then $f \circ g$ is intensive and lower semiidempotent.

Proof. From Theorem 7.3, we can see that $g$ is now an increasingly upper $f$-seminormal function of $Y^{\prime}$ to $X^{\prime}$. Moreover, we can note that $X^{\prime}$ is also reflexive. Therefore, by Theorem 9.1, $f \circ g$ is an extensive and upper semiidempotent operation on $Y^{\prime}$. Hence, by the corresponding definitions, it is clear $f \circ g$ is an intensive and lower semiidempotent operation on $Y$.

Corollary 10.2. If $f$ is an increasingly lower g-seminormal function of a reflexive goset $X$ to an arbitrary one $Y$ such that $g$ is increasing, then $g \circ f \circ g \leq g$.

Theorem 10.3. If $f$ is an increasingly upper g-seminormal function of an arbitrary goset $X$ to a reflexive one $Y$, then $g \leq g \circ f \circ g$.

Corollary 10.4. If $f$ is an increasingly $g$-normal function of a reflexive, antisymmetric goset $X$ to a reflexive one $Y$ such that $g$ is increasing, then $g=g \circ f \circ g$.

Theorem 10.5. If $f$ is an increasingly g-normal function of a transitive goset $X$ to a reflexive one $Y$, then $f \circ g$ is upper semiidempotent.

Corollary 10.6. If $f$ is an increasingly g-normal function of a proset $X$ to a reflexive goset $Y$, then $f \circ g$ is a semiinterior operation.

Theorem 10.7. If $f$ is an increasingly g-normal function of a reflexive goset $X$ to a transitive one $Y$, then $g$ is increasing and $g \circ f \circ g \leq g$.

Corollary 10.8. If $f$ is an increasingly g-normal function of a reflexive, antisymmetric goset $X$ to a proset $Y$, then $g=g \circ f \circ g$. 
Theorem 10.9. If $f$ is an increasingly g-normal function of a reflexive, antisymmetric goset $X$ to a reflexive one $Y$ such that $g$ is injective and either $g$ is increasing or $Y$ is transitive, then $f \circ g=\Delta_{Y}$, and thus $f$ is onto $Y$.

Theorem 10.10. If $f$ is an increasingly g-normal function of a reflexive, antisymmetric goset $X$ to a reflexive one $Y$ such that $g$ is onto $X$ and either $g$ is increasing or $Y$ is transitive, then $g \circ f=\Delta_{X}$, and thus $f$ is injective.

Theorem 10.11. If $f$ is an increasingly g-normal function of one proset $X$ to another $Y$, then $f \circ g$ is an interior operation on $Y$.

Theorem 10.12. If $f$ is an increasing function of an arbitrary goset $X$ to a transitive one $Y$ and $g$ is an arbitrary function of $Y$ to $X$ such that $f \circ g$ is intensive, then $f$ is increasingly lower g-seminormal.

Corollary 10.13. If $f$ is an increasing function $f$ on a reflexive goset $X$ to a transitive one $Y$ and $g$ is an arbitrary function of $Y$ to $X$, then $f \circ g$ is intensive if and only if $f$ is increasingly lower g-seminormal.

Now, combining Theorems 9.12 and 10.12, we can also state

Theorem 10.14. If $f$ is an increasing function of one transitive goset $X$ to another $Y$ and $g$ is an increasing function of $Y$ to $X$ such that the operation $g \circ f$ is extensive and the operation $f \circ g$ is intensive, then $f$ is increasingly g-normal.

Moreover, as an immediate consequence of Theorems 9.1, 9.7, 10.1, 10.7 and 10.14, we can also state

Theorem 10.15. For a function $f$ on one proset $X$ to another $Y$ and a function $g$ on $Y$ to $X$, the following assertions are equivalent:

(1) $f$ is increasingly g-normal,

(2) $f$ and $g$ are increasing, $g \circ f$ is extensive and $f \circ g$ is intensive.

Remark 10.16. Note that assertion (2) is just a dual of Ore's classical definition of Galois connexions [16].

Finally, we note that from Theorems 9.14 and 9.15, by using Theorem 7.3, we can immediately derive the following two theorems. 
Theorem 10.17. If $\varphi$ is an increasingly lower $\varphi$-seminormal operation on a reflexive goset $X$, then $\varphi$ is lower semiinvolutive.

Theorem 10.18. If $\varphi$ is an increasing, lower semiinvolutive operation on a transitive goset $X$, then $\varphi$ is increasingly lower $\varphi$-seminormal.

Hence, it is clear that in particular we also have

Corollary 10.19. A unary operation $\varphi$ on a proset $X$ is increasingly lower $\varphi$-seminormal if and only if it is increasing and lower semiinvolutive.

\section{Characterizations of Increasingly Seminormal Functions}

The following theorems and their corollaries have also been proved in [35].

Theorem 11.1. For a function $f$ of one goset $X$ to another $Y$ and a function $g$ of $Y$ to $X$, the following assertions are equivalent:

(1) $\mathrm{lb}(g(y)) \subseteq \operatorname{Int}_{f}(y)$ for all $y \in Y$,

(2) $f$ is an increasingly lower g-seminormal.

Theorem 11.2. For a function $f$ of one goset $X$ to another $Y$ and a function $g$ of $Y$ to $X$, the following assertions are equivalent:

(1) Int $_{f}(y) \subseteq \mathrm{lb}(g(y))$ for all $y \in Y$,

(2) $g(y) \in \mathrm{ub}\left(\operatorname{Int}_{f}(y)\right)$ for all $y \in Y$,

(3) $f$ is an increasingly upper g-seminormal.

Corollary 11.3. For a function $f$ of one goset $X$ to another $Y$ and a function $g$ of $Y$ to $X$, the following assertions are equivalent:

(1) $f$ is an increasingly g-normal, (2) $\operatorname{Int}_{f}(y)=\mathrm{lb}(g(y))$ for all $y \in Y$.

Theorem 11.4. If $f$ is an increasingly lower $g$-seminormal function of a reflexive goset $X$ to an arbitrary one $Y$, then for any $y \in Y$ we have

$$
g(y) \in \operatorname{Int}_{f}(y) .
$$


Corollary 11.5. If $f$ is an increasingly g-normal function of a reflexive goset $X$ to an arbitrary one $Y$, then for any $y \in Y$ we have
(1) $g(y) \in \max \left(\operatorname{Int}_{f}(y)\right)$,
(2) $g(y) \in \operatorname{Int}_{f}(y) \subseteq \mathrm{lb}(g(y))$.

Remark 11.6. Note that if in addition $X$ is antisymmetric, then by Theorem 3.5 we may write $g(y)=\max \left(\operatorname{Int}_{f}(y)\right)$ in assertion (1).

Theorem 11.7. If $f$ is an increasing function of an arbitrary goset $X$ to a transitive one $Y$ and $g$ is a function of $Y$ to $X$ such that

$$
g(y) \in \operatorname{Int}_{f}(y)
$$

for all $y \in Y$, then $f$ is increasingly lower g-seminormal.

Corollary 11.8. For an increasing function $f$ on a reflexive goset $X$ to a transitive one $Y$ and an arbitrary function $g$ of $Y$ to $X$, the following assertions are equivalent:

(1) $g(y) \in \operatorname{Int}_{f}(y)$ for all $y \in Y$,

(2) $f$ is increasingly lower g-seminormal.

Theorem 11.9. For a function $f$ of one proset to another $Y$ and a function $g$ of $Y$ to $X$, the following assertions are equivalent:

(1) $f$ is increasingly g-normal,

(2) $f$ is increasing and $g(y) \in \max \left(\operatorname{Int}_{f}(y)\right)$ for all $y \in Y$.

Remark 11.10. Note that if in particular $X$ is a poset, then by Theorem 3.5 we may write $g(y)=\max \left(\operatorname{Int}_{f}(y)\right)$ for all $y \in Y$ in assertion (2).

From the above results, we can immediately derive several characterizations of upper and lower semiinvolutive operations.

For instance, from Theorem 11.9, by using Theorems 9.16 and 10.9, we can immediately derive the following

Theorem 11.11. For an increasing operation $\varphi$ on proset $X$, the following assertions are equivalent:

(1) $\varphi(x) \in \max \left(\operatorname{Int}_{\varphi}(x)\right)$ for all $x \in X$,

(2) $\varphi$ is both upper and lower semiinvolutive. 
Remark 11.12. Note that if in particular $X$ is a poset, then in (2) we may simply write that $\varphi$ is involutive.

From the above results, by using the Axiom of Choice, we can also immediately derive several useful characterizations of increasingly upper and lower seminormal functions.

For instance, from Theorem 11.9 we can immediately derive the following

Theorem 11.13. For a function $f$ of one proset $X$ to another $Y$, the following assertions are equivalent:

(1) $f$ is increasingly normal,

(2) $f$ is increasing and $\max \left(\operatorname{Int}_{f}(y)\right) \neq \varnothing$ for all $y \in Y$.

Hence, it is clear that in particular we also have

Corollary 11.14. For a function $f$ of a max-complete proset $X$ to an arbitrary one $Y$, the following assertions are equivalent:

(1) $f$ is increasingly normal,

(2) $f$ is increasing and $F[x]$ is descending in $Y$.

Now, more specially we can also state

Corollary 11.15. For a function $f$ of a max-complete proset $X$ onto an arbitrary one $Y$, the following assertions are equivalent:
(1) $f$ is increasing,
(2) $f$ is increasingly normal.

\section{Some Further Characterizations of Increasingly Normal Functions}

In this section, we shall extend the results of [31, Section 7] to the present more general setting of increasingly normal functions.

For this, it is convenient to start with the following striking property of increasingly normal functions which also fails to hold for the increasing ones.

Theorem 12.1. If $f$ is an increasingly normal function of one goset $X$ to another $Y$, then for any $A \subseteq X$ we have

$$
f[\mathrm{lb}(\mathrm{ub}(A))] \subseteq \mathrm{lb}(\mathrm{ub}(f[A])) .
$$


Proof. If $y \in f[\operatorname{lb}(\mathrm{ub}(A))]$, then there exists $x \in \operatorname{lb}(\mathrm{ub}(A))$ such that $y=f(x)$. Moreover, if $v \in \operatorname{ub}(f[A])$, then for any $a \in A$ we have $f(a) \leq v$. Hence, by using that $f$ is increasingly $g$-normal, for some function $g$ of $Y$ to $X$, we can infer that $a \leq g(v)$. Therefore, $g(v) \in \mathrm{ub}(A)$, and thus because of $x \in \mathrm{lb}(\mathrm{ub}(A))$ we have $x \leq g(v)$. Hence, by using the increasing $g$-normality of $f$, we can infer that $f(x) \leq v$, and thus $y \leq v$. Therefore, $y \in \operatorname{lb}(\mathrm{ub}(f[A]))$ also holds.

From this theorem, by Theorem 3.3, it is clear that in particular we also have

Corollary 12.2. If $f$ is an increasingly normal function of one goset $X$ to another $Y$, then for any $A \subseteq X$ we have

$$
f[\sup (A)] \subseteq \operatorname{lb}(\operatorname{ub}(f[A])) .
$$

Hence, by using Theorem 6.14, we can immediately derive the following

Corollary 12.3. If $f$ is an increasingly normal function of one goset $X$ to another $Y$, then for any $y \in Y$ we have

$$
\max \left(\operatorname{Int}_{f}(y)\right)=\sup \left(\operatorname{Int}_{f}(y)\right) .
$$

However, it is now more important to note that from Theorem 12.1, by using Theorems 3.3 and 4.8 , we can also easily get the following

Theorem 12.4. If $f$ is an increasing, increasingly normal function of one goset $X$ to another $Y$, then for any $A \subseteq X$ we have

$$
f[\sup (A)] \subseteq \sup (f[A]) .
$$

Proof. By Theorems 3.3, 4.8 and 12.1, we have

$$
\begin{aligned}
f[\sup (A)] & =f[\mathrm{ub}(A) \cap \mathrm{lb}(\mathrm{ub}(A))] \subseteq f[\mathrm{ub}(A)] \cap f[\operatorname{lb}(\operatorname{ub}(A))] \\
& \subseteq \mathrm{ub}(f[A]) \cap \mathrm{bb}(\mathrm{ub}(f[A]))=\sup (f[A]) .
\end{aligned}
$$

Now, as an immediate consequence of Theorems 9.7 and 12.4, we can also state

Corollary 12.5. If $f$ is an increasingly normal function of a transitive goset $X$ to a reflexive one $Y$, then $f[\sup (A)] \subseteq \sup (f[A])$ for all $A \subseteq X$.

Hence, by Theorem 3.5, it is clear that in particular we also have 
Corollary 12.6. If $f$ is an increasingly normal function of a sup-complete, transitive goset $X$ to a reflexive, antisymmetric one $Y$, then $f(\sup (A))=\sup (f[A])$ for all $A \subseteq X$.

Now, to have some partial converses of the above results, we can also easily prove the following

Theorem 12.7. For a function $f$ of a sup-complete proset $X$ to an arbitrary one $Y$, the following assertions are equivalent:

(1) $f$ is increasingly normal,

(2) $f[\sup (A)] \subseteq \sup (f[A])$ for all $A \subseteq X$,

(3) $f$ is increasing and $\sup \left(\operatorname{Int}_{f}(y)\right) \subseteq \operatorname{Int}_{f}(y)$ for all $y \in Y$,

(4) $f$ is increasing and $\max \left(\operatorname{Int}_{f}(y)\right)=\sup \left(\operatorname{Int}_{f}(y)\right)$ for all $y \in Y$,

(5) $f$ is increasing and $f[\sup (A)] \subseteq \operatorname{lb}(\mathrm{ub}(f[A]))$ for all $A \subseteq X$,

(6) $f$ is increasing and $f[\mathrm{lb}(\mathrm{ub}(A))] \subseteq \mathrm{lb}(\mathrm{ub}(f[A]))$ for all $A \subseteq X$.

Proof. From Theorems 9.7 and 12.1, we can see that (1) implies (6). Moreover, from Theorem 3.3, we can see that $\sup (A) \subset \operatorname{lb}(\mathrm{ub}(A))$, and thus $f[\sup (A)] \subseteq f[\operatorname{lb}(\operatorname{ub}(A))]$. Therefore, (6) also implies (5).

Furthermore, from Theorem 6.14, we can see that (5) implies (4). Moreover, if (4) holds, then by the assumed sup-completeness of $X$ we have $\max \left(\operatorname{Int}_{f}(y)\right) \neq \varnothing$ for all $y \in Y$. Therefore, by Theorem 11.13, assertion (1) also holds.

On the other hand, from Corollary 12.5, we can see that (1) also implies (2). Moreover, if (2) holds, then from Corollary 4.9 and Theorem 3.3 we can see that (5) also holds. Finally, by Theorem 3.4, it is clear that (3) and (4) are also equivalent.

Now, by using Theorem 3.5, we can easily establish the following two corollaries of Theorem 12.7.

Corollary 12.8. For a function $f$ of a sup-complete poset $X$ to a proset $Y$, the following assertions are equivalent:

(1) $f$ is increasingly normal,

(2) $f(\sup (A)) \in \sup (f[A])$ for all $A \subseteq X$, 
(3) $f$ is increasing and $f(\sup (A)) \in \mathrm{lb}(\mathrm{ub}(f[A]))$ for all $A \subseteq X$.

Corollary 12.9. For a function $f$ of a sup-complete proset $X$ to a poset $Y$, the following assertions are equivalent:

(1) $f$ is increasingly normal,

(2) $f[\sup (A)]=\sup (f[A])$ for all $A \subseteq X$.

Remark 12.10. Note that, if in addition $X$ is also a poset, then by Theorem 3.5 we may again write $f(\sup (A))$ in place of $f[\sup (A)]$.

\section{Characterizations of Increasingly Semiregular Functions}

The following theorems and its corollaries have also been proved in [35].

Theorem 13.1. For a function $f$ of one goset $X$ to another $Y$ and a unary operation $\varphi$ on $X$, the following assertions are equivalent:

(1) $\mathrm{lb}(\varphi(x)) \subseteq \operatorname{Ord}_{f}^{-1}(x)$ for all $x \in X$,

(2) $f$ is increasingly lower $\varphi$-semiregular.

Theorem 13.2. For a function $f$ of one goset $X$ to another $Y$ and a unary operation $\varphi$ on $X$, the following assertions are equivalent:

(1) $\operatorname{Ord}_{f}^{-1}(x) \subseteq \mathrm{lb}(\varphi(x))$ for all $x \in X$,

(2) $\varphi(x) \in \operatorname{ub}\left(\operatorname{Ord}_{f}^{-1}(x)\right)$ for all $x \in X$,

(3) $f$ is increasingly upper $\varphi$-semiregular .

Corollary 13.3. For a function $f$ of one goset $X$ to another $Y$ and a unary operation $\varphi$ on $X$, the following assertions are equivalent:

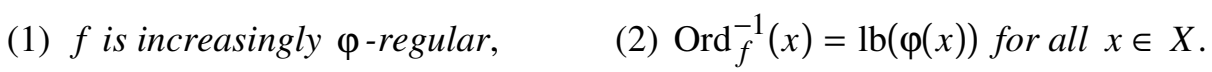

Theorem 13.4. If $f$ is an increasingly lower $\varphi$-semiregular function of a reflexive goset $X$ to an arbitrary one $Y$, then for any $x \in X$ we have

$$
\varphi(x) \in \operatorname{Ord}_{f}^{-1}(x)
$$


Corollary 13.5. If $f$ is an increasingly $\varphi$-regular function of a reflexive goset $X$ to an arbitrary one $Y$, then for any $x \in X$ we have
(1) $\varphi(x) \in \max \left(\operatorname{Ord}_{f}^{-1}(x)\right)$,
(2) $\varphi(x) \in \operatorname{Ord}_{f}^{-1}(x) \subseteq \operatorname{lb}(\varphi(x))$.

Remark 13.6. Note that if in addition $X$ is antisymmetric, then by Theorem 3.5 we may write $\varphi(x)=\max \left(\operatorname{Ord}_{f}^{-1}(x)\right)$ in assertion (2).

Theorem 13.7. If $f$ is an increasing function of an arbitrary goset $X$ to a transitive one $Y$ and $\varphi$ is a unary operation on $X$ such that

$$
\varphi(x) \in \operatorname{Ord}_{f}^{-1}(x)
$$

for all $x \in X$, then $f$ is increasingly lower $\varphi$-semiregular.

Corollary 13.8. For an increasing function $f$ of a reflexive goset $X$ to a transitive one $Y$ and a unary operation $\varphi$ on $X$, the following assertions are equivalent:

(1) $\varphi(x) \in \operatorname{Ord}_{f}^{-1}(x)$ for all $x \in X$,

(2) $f$ is increasingly lower $\varphi$-semiregular.

Theorem 13.9. For a function $f$ of one proset $X$ to another $Y$ and a unary operation $\varphi$ on $X$, the following assertions are equivalent:

(1) $f$ is increasingly $\varphi$-regular,

(2) $f$ is increasing and $\varphi(x) \in \max \left(\operatorname{Ord}_{f}^{-1}(x)\right)$ for all $x \in X$.

Remark 13.10. Note that if in particular $X$ is a poset, then by Theorem 3.5 we may write $\varphi(x)=\max \left(\operatorname{Ord}_{f}^{-1}(x)\right)$ in assertion (2).

From the above results, by using the Axiom of Choice, we can immediately derive several useful characterizations of increasingly lower and upper semiregular functions.

For instance, from Theorem 13.9, it is clear that we have the following

Theorem 13.11. For a function $f$ of one proset $X$ to another $Y$, the following assertions are equivalent: 
(1) $f$ is increasingly regular,

(2) $f$ is increasing and $\max \left(\operatorname{Ord}_{f}^{-1}(x)\right) \neq \varnothing$ for all $x \in X$.

Now, by using Corollary 7.9 and Theorems 13.11 and 11.13, we can also prove

Corollary 13.12. For a function $f$ of one proset $X$ onto another $Y$, the following assertions are equivalent:

(1) $f$ is increasingly regular, (2) $f$ is increasingly normal.

Proof. If (1) holds, then by Theorem 13.11 we can see that $f$ is increasing and $\max \left(\operatorname{Ord}_{f}^{-1}(x)\right) \neq \varnothing$ for all $x \in X$. Moreover, by using Theorem 6.9, we can see that

$$
\max \left(\operatorname{Int}_{f}(f(x))\right)=\max \left(\left(\operatorname{Int}_{f} \circ f\right)(x)\right)=\max \left(\operatorname{Ord}_{f}^{-1}(x)\right) \neq \varnothing
$$

for all $x \in X$. Hence, by using that $Y=f[X]$, we can already infer that $\max \left(\operatorname{Int}_{f}(y)\right) \neq \varnothing$ for all $y \in Y$. Thus, by Theorem 11.13, assertion (2) also holds. The converse implication (2) $\Rightarrow(1)$ is immediate from Corollary 7.9.

By using this corollary, from the results of Section 12 we can immediately derive some useful supremum properties of increasingly regular functions.

For instance, from Theorem 12.7, by using Corollary 13.12 and Theorem 6.9, we can immediately derive the following partial generalization of [31, Corollary 8.2].

Theorem 13.13. For a function $f$ of a sup-complete proset $X$ to an arbitrary one $Y$, under the notation $Z=f[X]$, the following assertions are equivalent:

(1) $f$ is increasingly regular,

(2) $f[\sup (A)] \subseteq \sup _{Z}(f[A])$ for all $A \subseteq X$,

(3) $f$ is increasing and $\sup \left(\operatorname{Ord}_{f}^{-1}(x)\right) \subseteq \operatorname{Ord}_{f}^{-1}(x)$ for all $x \in X$,

(4) $f$ is increasing and $\max \left(\operatorname{Ord}_{f}^{-1}(x)\right)=\sup \left(\operatorname{Ord}_{f}^{-1}(x)\right)$ for all $x \in X$.

Hence, by using Theorem 8.17 , we can immediately derive the following partial generalization of Theorem 5.10.

Corollary 13.14. For a unary operation $\varphi$ on a sup-complete proset $X$, under the 
notation $Y=\varphi[X]$, the following assertions are equivalent:

(1) $\varphi$ is a closure operation,

(2) $\varphi[\sup (A)] \subseteq \sup _{Y}(\varphi[A])$ for all $A \subseteq X$,

(3) $\varphi$ is increasing and $\sup \left(\operatorname{Ord}_{\varphi}^{-1}(x)\right) \subseteq \operatorname{Ord}_{\varphi}^{-1}(x)$ for all $x \in X$,

(4) $\varphi$ is increasing and $\max \left(\operatorname{Ord}_{\varphi}^{-1}(x)\right)=\sup \left(\operatorname{Ord}_{\varphi}^{-1}(x)\right)$ for all $x \in X$.

Remark 13.15. Note that if in particular $X$ is a sup-complete poset, then in (2) we may simply write $\varphi(\sup (A))=\sup _{Y}(\varphi[A])$.

\section{Directions to Some Further Reasonable Investigations}

Some results of this paper can also be generalized to relator spaces of the form $(X, Y)(\mathcal{R})=((X, Y), \mathcal{R})$, where $X$ and $Y$ are sets and $\mathcal{R}$ is a relator (family of relations) on $X$ to $Y$. (For the origins of these concepts, see [22] and the references therein.)

Note that relator spaces of the simpler type $X(\mathcal{R})=(X, X)(\mathcal{R})$ are already substantial generalizations of ordered sets [7] and uniform spaces [9]. However, they are insufficient to include context spaces [10, p. 17] (which are simple relator spaces of the form $(X, Y)(R)=(X, Y)(\{R\})$ where $R$ is a relation on $X$ to $Y)$, and also to naturally express continuity properties of functions and relations on one relator space to another $[26,37,50]$.

If $(X, Y)(\mathcal{R})$ and $(Z, W)(\mathcal{S})$ are relator spaces, and $\square$ is a direct unary operation for relators, then according to the ideas of [40, 39, 42] a relator $\mathcal{F}$ on $X$ to $Z$ may, for instance, be naturally called increasingly upper $\square-\mathcal{G}$-seminormal, for some relator $\mathcal{G}$ on $W$ to $Y$, if

$$
\left(\mathcal{S}^{\square} \circ \mathcal{F}^{\square}\right)^{\square} \subset\left(\left(\mathcal{G}^{\square}\right)^{-1} \circ \mathcal{R}^{\square}\right)^{\square}
$$

That is, the pair $(\mathcal{F}, \mathcal{G})$ is upper $\square$-semicontinuous by a straightforward generalization of [26, Definition 4.1] from relations to relators.

Here, $\square$ is a function of the class of all relator spaces such that, for any relator $\mathcal{R}$ 
on $X$ to $Y$, the value $\mathcal{R}^{\square}=\mathcal{R}^{\square} \square_{X Y}=\square((X, Y)(\mathcal{R}))$ is a relator on $X$ to $Y$. Moreover, the inversion and composition for relators are defined elementwise.

Note that the inversion -1 , defined by $\mathcal{R}^{-1}=\left\{R^{-1}: R \in \mathcal{R}\right\}$ for every relator $\mathcal{R}$ on $X$ to $Y$, is a nondirect unary operation for relators. While, the complementation $c$, defined by $\mathcal{R}^{c}=\left\{R^{c}: R \in \mathcal{R}\right\}$ for every relator $\mathcal{R}$ on $X$ to $Y$, is already a direct unary operation for relators.

Of course, if we restrict ourselves only to relator spaces of the simpler form $X(\mathcal{R})$, then -1 would be a direct operation for relators. Moreover, the modifications $\infty$ and $\partial$, defined by

$$
\mathcal{R}^{\infty}=\left\{R^{\infty}: R \in \mathcal{R}\right\} \quad \text { and } \quad \mathcal{R}^{\partial}=\left\{S \subseteq X^{2}: S^{\infty} \in \mathcal{R}\right\}
$$

for any relator $\mathcal{R}$ on $X$, would also be direct unary operations for relators. Concerning the latter operations, it is worth noticing that $\infty$ is an increasingly $\partial$-normal function, and thus $\partial \infty$ is a closure operation on the family of all relators on $X$.

However, it is now more important to note that, for instance, the proximal closure \#, defined by

$$
R^{\#}=\{S \subseteq X \times Y: \quad \forall A \subseteq X: \quad \exists R \in \mathcal{R}: \quad R[A] \subseteq S[A]\}
$$

for every relator $\mathcal{R}$ on $X$ to $Y$, and its dual $\boxplus=c \# c$ are also important direct unary operations for relators.

Namely, if $\mathcal{R}$ is a relator on $X$ to $Y$, then by defining

$$
\mathrm{Lb}_{\mathcal{R}}(B)=\{A \subset X: \quad \exists R \in \mathcal{R}: \quad A \times B \subset R\}
$$

and

$$
\operatorname{Int}_{\mathcal{R}}(B)=\{A \subset X: \quad \exists R \in \mathcal{R}: \quad R[A] \subset B\}
$$

for all $B \subseteq Y$, it can be shown that $\mathcal{S}=\mathcal{R}^{\#}\left(\mathcal{S}=\mathcal{R}^{\oplus}\right)$ is the largest relator on $X$ to $Y$ such that $\operatorname{Int}_{\mathcal{S}}=\operatorname{Int}_{\mathcal{R}}\left(\mathrm{Lb}_{\mathcal{S}}=\mathrm{Lb}_{\mathcal{R}}\right)$.

For this, it is enough to use only some basic facts on increasingly regular functions of one power set to another $[17,30]$, and the curious fact that $\operatorname{Lb}_{\mathcal{R}}=\operatorname{Int}_{\mathcal{R}^{\mathrm{c}}} \circ \mathcal{C}$, where 
$\mathcal{C}(B)=Y \backslash B$ for all $B \subseteq Y$. The latter equality, proved first in [27], establishes a similar connection between analysis and algebra as the famous Euler formula does between exponential and trigonometric functions [21, p. 227] .

From the results of [30], it has become completely clear that, to unitedly treat the several basic structures derived from relators [27] and their associated closure and modification operations [37], it is necessary to investigate first increasingly seminormal and semiregular functions of one power set to another. Therefore, these functions have to be studied at least three stages of generality. Firstly for posets, secondly for power sets, and thirdly for relator spaces.

To study increasingly normal and regular function of one power set $\mathcal{P}(X)$ to a goset $Y$, in an immediate continuation of this paper, for a function $F$ on a power set $\mathcal{P}(X)$ to a goset $Y$ we shall carefully investigate the set-valued functions $G_{F}$ and $\Phi_{F}$, defined by

$$
G_{F}(y)=\{x \in X: F(x) \leq y\} \text { and } \Phi_{F}(A)=\{x \in X: F(x) \leq F(A)\}
$$

for all $y \in Y$ and $A \subseteq X$. (Note that, here by identifying singletons with their elements, we have again written $x$ in place of $\{x\}$ for all $x \in X$.) Thus, we also have $\Phi_{F}=G_{F} \circ F$. Moreover, we may naturally look for some necessary and sufficient conditions in order that $F$ could be increasingly upper and lower $G_{F}$-seminormal and $\Phi_{F}$-semiregular, respectively.

\section{References}

[1] G. Birkhoff, Lattice Theory, Amer. Math. Soc. Colloq. Publ. 25, Providence, RI, 1967.

[2] T. S. Blyth and M. F. Janowitz, Residuation Theory, Pergamon Press, Oxford, 1972.

[3] Z. Boros and Á. Száz, Finite and conditional completeness properties of generalized ordered sets, Rostock. Math. Kolloq. 59 (2005), 75-86.

[4] Z. Boros and Á. Száz, Infimum and supremum completeness properties of ordered sets without axioms, An. St. Univ. Ovid. Constanta 16 (2008), 1-7.

[5] A. Brøndsted, Fixed points and partial orders, Proc. Amer. Math. Soc. 60 (1976), 365-366. https://doi.org/10.2307/2041175

[6] S. Buglyó and Á. Száz, A more important Galois connection between distance functions and inequality relations, Sci. Ser. A Math. Sci. (N.S.) 18 (2009), 17-38. 
[7] B. A. Davey and H. A. Priestley, Introduction to Lattices and Order, Cambridge University Press, Cambridge, 2002. https://doi.org/10.1017/CBO9780511809088

[8] K. Denecke, M. Erné and S. L. Wismath, eds., Galois Connections and Applications, Kluwer Academic Publisher, Dordrecht, 2004. https://doi.org/10.1007/978-1-4020-1898-5

[9] P. Fletcher and W. F. Lindgren, Quasi-Uniform Spaces, Marcel Dekker, New York, 1982.

[10] B. Ganter and R. Wille, Formal Concept Analysis, Springer-Verlag, Berlin, 1999. https://doi.org/10.1007/978-3-642-59830-2

[11] G. Gierz, K. H. Hofmann, K. Keimel, J. D. Lawson, M. Mislove and D. S. Scott, A Compendium of Continuous Lattices, Springer-Verlag, Berlin, 1980.

https://doi.org/10.1007/978-3-642-67678-9

[12] T. Glavosits, Generated preorders and equivalences, Acta Acad. Paed. Agriensis, Sect. Math. 29 (2002), 95-103.

[13] H. Kneser, Eine direkte Ableitung des Zirnschen lemmas aus dem Auswahlaxiom, Math. Z. 53 (1950), 110-113. https://doi.org/10.1007/BF01162404

[14] J. Mala and Á. Száz, Modifications of relators, Acta Math. Hungar. 77 (1997), 69-81. https://doi.org/10.1023/A:1006583622770

[15] J. Niederle, A useful fixpoint theorem, Rend. Circ. Mat. Palermo, II. Ser. 47 (1998), 463464. https://doi.org/10.1007/BF02851392

[16] O. Ore, Galois connexions, Trans. Amer. Math. Soc. 55 (1944), 493-513. https://doi.org/10.1090/S0002-9947-1944-0010555-7

[17] G. Pataki, On the extensions, refinements and modifications of relators, Math. Balk. 15 (2001), 155-186.

[18] W. J. Pervin, Quasi-uniformization of topological spaces, Math. Ann. 147 (1962), 316317. https://doi.org/10.1007/BF01440953

[19] Cs. Rakaczki and Á. Száz, Semicontinuity and closedness properties of relations in relator spaces, Mathematica (Cluj) 45 (2003), 73-92.

[20] J. Schmidt, Bieträge zur Filtertheorie II, Math. Nachr. 10 (1953), 197-232. https://doi.org/10.1002/mana.19530100309

[21] K. R. Stromberg, An Introduction to Classical Real Analysis, Wadsworth, Belmont, California, 1981. 
[22] Á. Száz, Basic tools and mild continuities in relator spaces, Acta Math. Hungar. 50 (1987), 177-201. https://doi.org/10.1007/BF01903935

[23] Á. Száz, Structures derivable from relators, Singularité 3 (1992), 14-30.

[24] Á. Száz, Refinements of relators, Tech. Rep., Inst. Math., Univ. Debrecen 76 (1993), $19 \mathrm{pp}$.

[25] Á. Száz, A Galois connection between distance functions and inequality relations, Math. Bohem. 127 (2002), 437-448.

[26] Á. Száz, Somewhat continuity in a unified framework for continuities of relations, Tatra Mt. Math. Publ. 24 (2002), 41-56.

[27] Á. Száz, Upper and lower bounds in relator spaces, Serdica Math. J. 29 (2003), $239-270$.

[28] Á. Száz, Lower and upper bounds in ordered sets without axioms, Tech. Rep., Inst. Math., Univ. Debrecen 2004/1, 11 pp.

[29] Á. Száz, The importance of reflexivity, transitivity, antisymmetry and totality in generalized ordered sets, Tech. Rep., Inst. Math., Univ. Debrecen 2004/2, 15 pp.

[30] Á. Száz, Galois-type connections on power sets and their applications to relators, Tech. Rep., Inst. Math., Univ. Debrecen 2005/2, 38 pp.

[31] Á. Száz, Supremum properties of Galois-type connections, Comment. Math. Univ. Carolin. 47 (2006), 569-583.

[32] Á. Száz, Minimal structures, generalized topologies, and ascending systems should not be studied without generalized uniformities, Filomat 21 (2007), 87-97. https://doi.org/10.2298/FIL0701087S

[33] Á. Száz, Galois type connections and closure operations on preordered sets, Acta Math. Univ. Comen. 78 (2009), 1-21.

[34] Á. Száz, Galois-type connections and continuities of pairs of relations, J. Int. Math. Virt. Inst. 2 (2012), 39-66.

[35] Á. Száz, Galois and Pataki connections revisited, Tech. Rep., Inst. Math., Univ. Debrecen 2013/3, 20 pp.

[36] Á. Száz, Inclusions for compositions and box products of relations, J. Int. Math. Virt. Inst. 3 (2013), 97-125.

[37] Á. Száz, Lower semicontinuity properties of relations in relator spaces, Adv. Stud. Contemp. Math. (Kyungshang) 23 (2013), 107-158. 
[38] Á. Száz, A particular Galois connection between relations and set functions, Acta Univ. Sapientiae Math. 6 (2014), 73-91. https://doi.org/10.2478/ausm-2014-0019

[39] Á. Száz, Generalizations of Galois and Pataki connections to relator spaces, J. Int. Math. Virtual Inst. 4 (2014), 43-75.

[40] Á. Száz, Basic tools, increasing functions, and closure operations in generalized ordered sets, in: P. M. Pardalos and Th. M. Rassias, eds., Contributions in Mathematics and Engineering: In Honor of Constantion Caratheodory, Springer, 2016, pp. 551-616. https://doi.org/10.1007/978-3-319-31317-7_28

[41] Á. Száz, A natural Galois connection between generalized norms and metrics, Acta Univ. Sapientiae Math. 9 (2017), 360-373. https://doi.org/10.1515/ausm-2017-0027

[42] Á. Száz, Four general continuity properties, for pairs of functions, relations and relators, whose particular cases could be investigated by hundreds of mathematicians, Tech. Rep., Inst. Math., Univ. Debrecen 2017/1, 17 pp.

[43] Á. Száz, Relationships between inclusions for relations and inequalities for corelations, Math. Pannon. 26 (2017-2018), 15-31.

[44] Á. Száz, The closure-interior Galois connection and its applications to relational equations and inclusions, J. Int. Math. Virt. Inst. 8 (2018), 181-224.

[45] Á. Száz, A unifying framework for studying continuity, increasingness, and Galois connections, MathLab J. 1 (2018), 154-173.

[46] Á. Száz, Corelations are more powerful tools than relations, in: Th. M. Rassias, ed., Applications of Nonlinear Analysis, Springer Optimization and Its Applications 134 (2018), 711-779. https://doi.org/10.1007/978-3-319-89815-5_25

[47] Á. Száz and J. Túri, Comparisons and compositions of Galois-type connections, Miskolc Math. Notes 7 (2006), 189-203. https://doi.org/10.18514/MMN.2006.144

[48] A. Weil, Sur les espaces á structure uniforme et sur la topologie générale, Actual. Sci. Ind. 551, Herman and Cie, Paris, 1937.

[49] W. Xia, Galois connections and formal concept analysis, Demonstratio Math. 27 (1994), 751-767. https://doi.org/10.1515/dema-1994-3-419

[50] A. Zakaria and Á. Száz, Mild continuity properties of relations and relators in relator spaces, in: P. M. Pardalos and Th. M. Rassias, eds., Essays in Mathematics and its Applications: In Honor of Vladimir Arnold, Springer, 2016, pp. 439-511. https://doi.org/10.1007/978-3-319-31338-2_16 